\title{
eJRIEPS
}

Ejournal de la recherche sur l'intervention en éducation physique et sport

$31 \mid 2014$

Varia

\section{Repenser l'accompagnement des enseignants novices pour accroitre leur satisfaction professionnelle}

Amathieu Jérôme et Chaliès Sébastien

\section{(2) OpenEdition}

Édition électronique

URL : http://journals.openedition.org/ejrieps/2184

DOI : 10.4000/ejrieps.2184

ISSN : 2105-0821

Éditeur

ELLIADD

Référence électronique

Amathieu Jérôme et Chaliès Sébastien, «Repenser l'accompagnement des enseignants novices pour accroitre leur satisfaction professionnelle », eJRIEPS [En ligne], 31 | 2014, mis en ligne le 01 janvier 2014, consulté le 03 octobre 2019. URL : http://journals.openedition.org/ejrieps/2184 ; DOI : 10.4000/ ejrieps. 2184

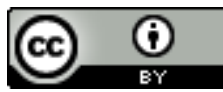

La revue eJRIEPS est mise à disposition selon les termes de la Creative Commons Attribution 4.0 International License. 
eJRIEPS 31 janvier 2014

Repenser l'accompagnement des enseignants novices pour accroitre leur satisfaction professionnelle

Amathieu Jérôme \& Chaliès Sébastien

Laboratoire UMR EFTS - Equipe CReFoDeP

Université de Toulouse II - IUFM de Midi-Pyrénées

Résumé

L'augmentation de la charge de travail des enseignants novices (EN) et les problèmes de santé au travail qu'elle engendre sont significatifs des difficultés de l'entrée dans le métier des EN. Pour y répondre, des aménagements de la formation sont proposés sans que pour autant leurs retombées soient réellement évaluées. L'objet de cette étude, menée à partir d'une théorie de l'action collective (Wittgenstein, 1996), se situe à ce niveau. En prenant comme dispositif support un aménagement de formation, cette étude montre principalement que sa mise en œuvre n'a que peu d'impact sur la santé au travail des EN. Deux résultats majeurs étayent cette conclusion. Tout d'abord, les formateurs procèdent rarement à un enseignement ostensif de nouvelles règles relatives à ce qui pourrait être réalisé en classe ainsi qu'aux résultats qui pourraient en être attendus. Autrement dit, les EN ne sont pas placés en situation de " mieux faire " (Clot, 2010) leur travail et par-là même d'en être satisfaits. Sans ces règles, les EN ne peuvent en effet interpréter, juger et in fine s'adapter aux diverses expériences vécues en s'engageant dans des actions singulières et attendues au sein de la communauté professionnelle. D'autre part, les formateurs ne considèrent jamais la situation de classe comme une situation de formation potentielle. Non aménagée, cette dernière place régulièrement les EN en situation d'insatisfaction professionnelle aboutissant à du stress au travail.

Mots clés : satisfaction au travail, formation initiale, enseignants novices (EN)

\section{Introduction}

Dans la littérature scientifique du domaine, la satisfaction professionnelle est généralement assimilée au fait que les travailleurs aiment ou non leur travail et, en le réalisant, y développent un sentiment de bien-être (Weiss, 2002). Plus spécifiquement, elle est considérée comme la résultante de la réalisation par les travailleurs de diverses 


\section{eJRIEPS 31 janvier 2014}

aspirations individuelles au travail (Fabra \& Camison, 2009). Ainsi, Francès (1995, p.12) précise que la satisfaction professionnelle implique «non seulement des résultats attendus affectés de leurs valences respectives, mais une confrontation de ceux-ci avec les résultats obtenus ". Autrement dit, la satisfaction professionnelle résulte des différences entre les attentes des enseignants et ce qu'ils rencontrent effectivement lorsqu'ils enseignent.

L'intérêt accordé à la satisfaction est d'autant plus important qu'elle possède une influence sur la santé des travailleurs et des enseignants en particulier. En cas d'insatisfaction professionnelle marquée, les enseignants présentent des troubles plus ou moins sévères de détresse psychologique, tels que l'anxiété, le stress voire la dépression (Demirtas, 2010). Ces troubles sont d'autant plus significatifs qu'ils peuvent dans certaines circonstances engendrer une situation d'épuisement psychologique et physique, qui conduit les enseignants à arrêter momentanément leur travail voire à l'abandonner (Skaalvik \& Skaalvik, 2010). Inversement, des travaux montrent que l'accroissement de la satisfaction professionnelle des enseignants améliore leur santé notamment psychologique (Moè, Pazzaglia \& Ronconi, 2010). Davantage satisfaits de leur travail, les enseignants développent progressivement un sentiment d'efficacité plus marqué et, en retour, réduisent leur stress (Kyriacou, 2001). Complémentairement, ils s'engagent de façon plus prononcée et enthousiaste dans leur travail auprès de leurs élèves (Shen, Leslie, Spybrook \& Ma, 2011).

Comment alors influencer la satisfaction pour améliorer la santé au travail ? La satisfaction professionnelle est usuellement placée sous l'influence de différents facteurs. Parmi les modèles cherchant à rendre compte de ces facteurs, celui proposé par Duffy \& Lent (2009) étudie l'influence de cinq facteurs interdépendants: le type de personnalité du travailleur, la qualité du but poursuivi, les aides à dispositions pour atteindre le but poursuivi, les conditions humaines et matérielles de travail, et le sentiment d'efficacité personnelle. Ces deux derniers facteurs possèdent une influence particulièrement significative sur la satisfaction professionnelle des enseignants.

La satisfaction professionnelle des enseignants est tout d'abord influencée par leurs conditions humaines et matérielles d'enseignement, c'est-à-dire par les conditions de travail offertes par leur établissement scolaire (Mtika \& Gates, 2011). Plus le climat leur apparait comme propice, plus leur satisfaction est accentuée. Parmi les conditions constitutives d'un climat favorable, il est possible de relever : (i) la possibilité qui leur est offerte de participer à la gouvernance de l'établissement et par-là même d'y développer un 


\section{eJRIEPS 31 janvier 2014}

véritable sentiment d'appartenance (Smylie, 1994), (ii) la reconnaissance de leur travail par leur hiérarchie, leurs collègues les parents et leurs élèves (Dinham, 1995), (iii) le caractère adapté de leur charge de travail (Liu \& Ramsey, 2008), (iv) le degré d'autonomie qui leur est accordé dans la réalisation de leur enseignement au quotidien (Skaalvik et al., 2010) et (v) les possibilités d'interagir professionnellement avec d'autres enseignants, voire d'engager des projets collectifs (Leung, Siu \& Spector, 2000).

La satisfaction professionnelle est par ailleurs déterminée par le sentiment d'efficacité personnelle des enseignants lors de la réalisation de leur travail (Caprara, Barbaranelli, Steca \& Malone, 2006). II existe en effet la possibilité de tracer un lien de causalité entre l'efficacité personnelle perçue au travail par les enseignants et leur satisfaction. Ainsi, plus ils se sentent efficaces au travail, plus les enseignants s'en trouvent satisfaits (Moe et al., 2010). Individuelle mais aussi collective, la satisfaction professionnelle est dépendante du sentiment d'efficacité que chaque enseignant s'attribue mais aussi qu'il attribue à l'établissement dans lequel il travaille (Judge, Thoresen, Bono \& Patton, 2001).

Alors que de nombreux travaux ont été réalisés pour étudier l'influence de ces deux facteurs sur la satisfaction professionnelle, il est assez surprenant de relever que rares sont au contraire ceux ayant essayé de mettre en tension cette dernière avec les aides mises à disposition des enseignants. La relation directe entre les circonstances de formation et la satisfaction des EN au travail semble négligée. Parmi les quelques travaux ayant interrogé ce lien, celui mené par Bullough, Bullough \& Mayes (2006) conclut sur les bénéfices d'un soutien émotionnel $\mathrm{du}$ mentor pour accroitre la satisfaction des enseignants novices. Dans le même ordre d'idées, Nir \& Bogler (2008) montrent tout l'intérêt de contenus de formation directement exploitables par les formés dans leur travail. Ce travail consiste donc à étudier les circonstances dans lesquelles les activités de formation menées par les formateurs ont une influence sur la satisfaction professionnelle des formés.

\section{Cadre théorique}

Dans cette étude, le principe de l'alternance entre situation de formation au travail, autrement dit de formation au travail d'enseignement à l'université dans cette étude, et situation de travail effective a été conceptualisé à partir de postulats empruntés à une théorie générale de l'apprentissage (Berducci, 2004; Nelson, 2008) et de l'action collective (Wittgenstein, 2004). Trois hypothèses empruntées à cette conceptualisation (pour plus de détails : Bertone, Chaliès \& Clot, 2009) ont été retenues. 


\section{eJRIEPS 31 janvier 2014}

2.1. Première hypothèse : Travailler et se former au travail nécessitent de s'engager dans un double régime de réflexivité

Dans la lecture théorique proposée, travailler revient à mener des actions gouvernées par des règles et/ou à réaliser des actions qui sont le suivi de règles. Ces règles sont des expériences situées normatives acceptées par la communauté professionnelle considérées (Wittgenstein, 2004). Autrement dit, ces règles n'ont d'autorité que si le travailleur décide de les suivre ou s'il est encouragé, par exemple par des formateurs, à les suivre (Descombes, 2004). Lorsqu'il travaille, chaque acteur s'engage plus précisément dans un double registre de réflexivité par rapport à ces règles (Ogien, 2007). Dans certaines situations professionnelles, il est engagé dans un régime de réflexivité de l'ordre de l'inhérence. II mène alors des " actions gouvernées" par les règles (Ogien, 2007). II agit donc selon ces dernières qui, à l'instant considéré lui sont soit préconscientes et potentiellement dicibles parce qu'apprises lors d'une formation réflexive antérieure, soit non conscientes et par-là même non dicibles. Dans un grand nombre de situations de travail, le travailleur réalise en effet des actions " gouvernées » par les règles dont l'apprentissage s'est fait implicitement (Lave \& Wenger, 1991) par l'intermédiaire d'interactions non verbales et/ou d'alignements informels avec la pratique des autres membres (Rogoff, Matusov \& White, 1996) au cours de sa participation aux pratiques professionnelles constitutives de la communauté. Dans d'autres situations professionnelles, le travailleur est engagé dans un régime de réflexivité de l'ordre de l'appréhension de l'inhérence : il réalise alors des actions qui sont le « suivi » de règles (Ogien, 2007). II agit donc en conformité avec ces dernières qui, à l'instant considéré, sont conscientes et dicibles parce qu'apprises lors d'une formation réflexive antérieure au cours de laquelle il a appris avec l'aide d'un ou plusieurs formateurs à les rendre intelligibles par l'intermédiaire de réélaborations discursives. Le principe de formation par alternance entre formation au travail d'enseignement et travail d'enseignement à proprement parler en établissement scolaire étant principalement fondé à partir de ce second régime de réflexivité, nous le détaillons ci-dessous.

2.2. Deuxième hypothèse : la formation professionnelle des enseignants novices repose sur l'apprentissage puis l'interprétation de règles par les enseignants novices

La possibilité d'un engagement des enseignants novices dans une pratique réflexive à propos de leur travail nécessite un apprentissage préalable de règles leur permettant de signifier correctement les expériences professionnelles qu'ils ont pu vivre, c'est-à-dire 


\section{eJRIEPS 31 janvier 2014}

comme il est attendu de le faire au sein de la communauté professionnelle considérée. Cet apprentissage nécessite l'engagement des formateurs dans une activité "d'enseignement ostensif " (Wittgenstein, 2004) par laquelle ils fondent la signification des expériences professionnelles considérées comme exemplaires. Pour ce faire, ils dressent ostensivement pour chacune de ces expériences enseignées un «lien de signification " (Bertone et al., 2009) entre, (i) l'expérience langagière visant à la nommer, (ii) les exemples décrits et/ou montrés en correspondance autrement appelés éléments d'étayage de la règle et (iii) les résultats qui y sont usuellement associés dans la communauté. Ces liens de significations enseignés, et donc initialement non construits par une pratique réflexive autonome, sont ensuite de véritables expériences "mètre étalon " (Williams, 2002) lorsque les EN agissent dans de nouvelles situations de travail ou de formation au travail. Ces derniers s'appuient alors en effet sur ces échantillons d'expériences exemplaires ostensiblement montrés pour signifier et juger les événements rencontrés, voire essayer de s'y adapter. L'idée d'un échantillonnage de l'expérience de travail pendant la formation peut être rattachée à celle de l'existence d'une sorte de " grammaire sociale » du métier (Laugier \& Chauviré, 2006), que possèdent et mobilisent les membres d'une communauté professionnelle donnée. Cette grammaire de l'expérience de travail peut faire l'objet d'un enseignement par les formateurs et d'une familiarisation pour les EN qui passe par des activités multiples telles que, entre autres, la description des faits observables ou à observer, l'identification « d'aspects " (Chauviré, 2010) saillants à considérer ou encore l'introduction de préférences perceptives rendant «parlantes » et non équivalentes les circonstances vécues dans l'exercice du métier (Narboux, 2003).

Les formateurs ne peuvent toutefois se suffire de cette activité d'enseignement. Ils doivent ensuite s'engager dans une activité d'accompagnement des premiers suivis par les EN des règles préalablement enseignées dans de nouvelles situations de formation ou de travail en classe. Par cet accompagnement, ils visent à rendre possible mais aussi et surtout à faire en sorte que ces premiers suivis aboutissent aux résultats attendus y étant usuellement associés. Ce n'est en effet qu'au travers du constat de ces résultats attendus que les EN peuvent associer consubstantiellement une intention professionnelle aux règles enseignées (Cash, 2009). Autrement dit, suivant correctement les règles préalablement enseignées et constatant les résultats qui leur sont usuellement associés dans la communauté professionnelle, les EN finalisent leur apprentissage de la règle considérée et peuvent alors se développer à partir de celles-ci (Nelson, 2008). Lors de ces premiers suivis, les formateurs «contrôlent» (Nelson, 2008) la conformité des premiers 


\section{eJRIEPS 31 janvier 2014}

suivis des règles enseignées par les formés et s'engagent si nécessaire dans une activité d'« explications ostensives » (Davis, 2009). Ils s'efforcent alors de multiplier les exemples décrits et/ou montrés pouvant être associés aux règles enseignées de sorte de lever les éventuelles incompréhensions ou mésinterprétations des enseignants novices et leur permettre, au final, de les suivre seuls et de façon acceptable, c'est-à-dire comme convenu dans la communauté professionnelle.

Lorsque les règles sont apprises, leurs suivis par les EN dans de nouvelles situations de travail ou de formation nécessitent leur interprétation (Winch, 2009). Ces suivis renvoient en effet à un usage extensif des liens de signification construits et appris sur la base de l'identification par les formés d'un "air de famille " (Wittgenstein, 2004) entre les circonstances constitutives de la situation en cours et celles constitutives des situations dans lesquelles les règles ont été apprises. A partir des liens de signification appris, les EN ordonnent alors finalement un réseau complexe de ressemblances, comprennent la nouvelle situation en cours, et in fine parviennent à produire des activités conformes aux attentes de la communauté, tout en s'émancipant progressivement de son contrôle. Ces situations de développement ne sont donc plus l'occasion pour les formateurs de contrôler le caractère adéquat ou inadéquat des significations associées par les EN aux événements rencontrés. Elles leur permettent plutôt d'aider ces derniers à s'engager dans des suivis interprétés des règles apprises, c'est-à-dire de les aider à en négocier la signification pour mieux en user.

2.3. Troisième hypothèse : la formation accroit la satisfaction en permettant aux EN d'obtenir par leur activité des résultats attendus

Dans la lecture théorique adoptée, le postulat développé en clinique de l'activité selon lequel « le travail bien fait » est « le ressort de la santé au travail » (Clot, 2010, p. 21) a été retenu comme un point d'ancrage compatible et complémentaire. La satisfaction professionnelle a en effet été considérée comme directement corrélée à la justesse du travail du point de vue de ceux qui le réalisent, c'est-à-dire corrélée à une «authentification rétrospective " (Chauviré, 2010) par les EN de ce qui était attendu. Autrement dit, aboutissant aux résultats attendus lors des suivis des règles considérées, les EN s'en trouvent finalement satisfaits. Ce constat peut relever de deux types de circonstances de formation. Tout d'abord, la satisfaction des EN peut être alimentée par les situations de formation concourant à l'apprentissage de nouvelles règles. La satisfaction est alors consubstantielle au constat par les EN des résultats attendus suite à leurs premiers suivis de toute règle préalablement enseignée mais non encore apprise. 


\section{eJRIEPS 31 janvier 2014}

D'autre part, la satisfaction des EN peut être alimentée par les situations de formation concourant à leurs nouveaux usages des règles préalablement apprises. En élargissant les circonstances dans lesquelles il est possible de "voir » ou "faire jouer » les règles apprises, les EN les ajustent en effet au cours de leurs suivis jusqu'à aboutir à ce qu'ils attendent en termes de résultats. C'est alors qu'ils se trouvent consubstantiellement satisfaits de leur activité.

\section{Méthode}

3.1. Dispositif de formation support à l'étude

Le dispositif de formation support à cette étude était celui de l'« Aide au stagiaire en grande difficulté " adressé aux EN considérés comme en " grande difficulté 》 dans leur travail. Cette difficulté était repérée suite aux visites d'évaluation formative effectuées en milieu de formation par, d'une part, la dyade constituée d'un Formateur Universitaire (FU) et d'un Tuteur ( $T$ ) et, d'autre part, un membre du corps d'inspection. Complémentaire aux dispositifs de formation usuels, ce dispositif était constitué de deux volets. Un premier volet était mené sous la forme de plusieurs séquences de formation dont l'objet principal était la "Prise en main des élèves ". Les formateurs avaient en effet identifié que c'était là la difficulté la plus significative des EN, c'est-à-dire la difficulté à partir de laquelle en découlaient d'autres. En conséquence, ils avaient donc décidé au sein du dispositif d'aide d'aider prioritairement les EN à construire des conditions sereines d'enseignement et d'apprentissage au sein de leur classe afin qu'ils puissent ensuite s'engager dans les diverses activités professionnelles attendues telles que la présentation de situation, l'explication de contenus disciplinaires ou l'accompagnement de l'apprentissage des élèves. Un second volet était effectué en établissement scolaire. C'est sur ce volet de la formation que l'étude a été réalisée. II était mené sous la forme d'une série de visites formatives impliquant la $T$ ayant la responsabilité de l'EN et une FU. Ces visites se structuraient autour de deux temps successifs: une leçon menée par l'EN avec sa classe sous l'observation conjointe du T et du FU, puis d'un temps de formation collectif de type entretien de conseil pédagogique impliquant l'ensemble des acteurs de la triade. En fin de leçon, le T et le FU faisaient un point sur leurs observations respectives en rapport avec l'objet de formation, à savoir la prise en main des élèves. L'ensemble des difficultés communément identifiées étaient sélectionnées comme objet de discussion avec l'EN. Lors de l'entretien de conseil pédagogique, ces difficultés étaient ensuite successivement abordées. Pour chacune d'entre elle, le FU avait la responsabilité de la décrire à l'EN et 


\section{eJRIEPS 31 janvier 2014}

d'y apporter des éléments de réponse potentiels. Après avoir invité l'EN à discuter de la difficulté sélectionnée et des éléments de réponse avancés pour y répondre, le FU invitait le T à aider l'EN à envisager la réponse la plus adaptée compte tenu des circonstances de classe et à anticiper sa mise en œuvre lors de la prochaine leçon.

\subsection{Participants}

Cette recherche a été menée avec deux triades de formation volontaires. Ces triades étaient constituées d'une EN, d'une tutrice et d'une FU. Agées de 24 et 26 ans, les EN étaient des PLC2 rattachées à l'IUFM de Midi-Pyrénées. Dans cette étude, les EN étaient des enseignants stagiaires en formation professionnelle initiale par alternance entre d'une part leur IUFM de rattachement et d'autre part leur établissement scolaire d'affectation. Elles avaient pour la première fois la responsabilité de leurs propres classes pendant une année scolaire. Au moment de la recherche, leur expérience en enseignement était de 48 journées en établissement scolaire. Les deux $T$ étaient à la fois considérées comme des enseignantes expérimentées (respectivement : 16 et 22 années d'expérience) mais aussi comme des T expérimentées (cinq années d'expérience chacune dans cette fonction). Les deux FU étaient des enseignantes du second degré exerçant à l'IUFM. Elles avaient toutes les deux une expérience d'enseignante (respectivement 14 et 18 années) et de T (respectivement 4 et 6 années).

\subsection{Recueil des données}

Pour réaliser cette étude, deux catégories de données ont été recueillies puis retranscrites sous forme de verbatim. Des données d'enregistrement ont été recueillies pendant les entretiens de conseil pédagogique menés par les EN et leurs formateurs à la suite de la leçon observée. Des données d'autoconfrontation ont aussi été recueillies. Pour ce faire, des entretiens d'autoconfrontation (EAC) ont également été menés avec les EN et leurs formateurs sur la base des données d'enregistrement des entretiens de conseil pédagogique. Ces EAC ont été conduits de façon à pouvoir reconstituer a posteriori les règles suivies par les acteurs pendant l'entretien de conseil pédagogique. Par un questionnement semi structuré, le chercheur a cherché à recueillir le plus grand nombre d'éléments possibles permettant de reconstituer a posteriori les règles suivies par l'acteur autoconfronté. II a donc incité l'acteur interviewé à l'instruire sur les significations à attribuer aux actions observées et aux jugements pouvant y être associés (par exemple : "Qu'est-ce que tu réalises comme action à ce moment-là ? ; "Qu'est-ce que tu penses de ton intervention à ce moment de classe ?»). En l'invitant à préciser ses propos (par exemple : "Je ne comprends pas pourquoi tu considères cette action comme 


\section{eJRIEPS 31 janvier 2014}

intéressante, peux-tu reprendre? "), ou jouant sur la controverse en plaçant l'acteur interviewé face à des contradictions apparentes (par exemple: "Ce qui est étonnant, c'est que tu dis que cet élève te dérange mais en même temps tu ne le reprends pas lorsqu'il perturbe la classe ? "), le chercheur a invité ensuite l'acteur à étayer les jugements préalablement portés sur les actions signifiées. Enfin, il a invité l'acteur à préciser les résultats attendus quant aux actions observées (par exemple : «Et là, tu t'attends à quoi lorsque tu interpelles cet élève ?»).

\subsection{Traitement des données}

Pour identifier et formaliser les règles apprises et/ou suivies par les différents acteurs au cours des différentes séquences du dispositif, nous avons adopté et aménagée la procédure proposée par Chaliès, Bertone, Flavier \& Durand (2008). Les données recueillies ont ainsi été traitées en cinq étapes successives:

(I) L'ensemble des verbalisations des acteurs autoconfrontés recueillies lors des entretiens d'autoconfrontation a été retranscrit verbatim. Retranscrits, ces verbatim ont ensuite été découpés en unités d'interaction. Ces unités ont été délimitées à partir de l'étiquetage langagier (autrement dit l'objet de la signification) attribué par l'acteur autoconfronté aux événements visionnés. Une nouvelle unité d'interaction a été créée à chaque fois que cet étiquetage attribué par l'acteur changeait ;

(ii) Pour chaque unité d'interaction, les éléments d'étayage associés par l'acteur autoconfronté à l'étiquetage considéré ont été ensuite identifiés. Par convention, ces éléments d'étayage correspondaient à l'ensemble des circonstances évoquées par l'acteur et associées à l'étiquetage considéré. Autrement dit, ces éléments d'étayage correspondaient à l'ensemble des circonstances évoquées pour expliquer au chercheur la façon de s'y prendre pour signifier de la même façon que lui, c'est-à-dire en suivant la même règle, les événements de la situation de formation de classe ou de formation visionnés.

(iii) Pour chaque unité d'interaction, la règle suivie par l'acteur pour signifier ce qu'il a fait et en juger a ensuite été formalisée. Par convention, chaque règle a été étiquetée à partir d'un travail de rassemblement (a) de l'étiquetage attribué par l'acteur, (b) de l'ensemble des circonstances associées par l'acteur à l'étiquetage et (c) des résultats constatés et/ou attendus. Une fois ce rassemblement des différents éléments constitutifs de la règle effectué, le chercheur procédait à sa formalisation. Dans sa forme, chaque règle était formalisée et présentée comme suit: ["Etiquetage " vaut dans les circonstances où "ensemble des circonstances associées à l'étiquetage » ce qui obtient 


\section{eJRIEPS 31 janvier 2014}

comme résultat "ensemble des résultats constatés et lou attendus"]. Afin de minimiser les interprétations du chercheur, chacune des règles a été étiquetée au plus près du vocabulaire des acteurs ;

(iv) Pour chaque unité d'interaction, la satisfaction professionnelle de l'acteur autoconfronté a été étudiée. II a été considéré que l'acteur était satisfait lorsque, suivant la règle qui lui avait été initialement enseignée, il parvenait à en constater les résultats attendus. Autrement dit, l'acteur a été considéré comme satisfait de ce qu'il faisait lorsque une adéquation pouvait être établie entre les résultats potentiellement attendus suite au suivi de la règle et ceux effectivement constatés ;

(v) La validité des résultats obtenus a été testée. L'ensemble du corpus a en effet été analysé de façon séparée par deux chercheurs. Tous les résultats obtenus ont ensuite été comparés et discutés par les deux chercheurs jusqu'à l'obtention d'un accord. En cas de désaccord, ils ont été rejetés ;

(vi) Une fois le traitement validé, chacune des règles formalisées a été interrogée du point de vue de ses différents suivis sur l'ensemble du dispositif longitudinal de formation. Pour ce faire, les chercheurs essayaient alors de repérer :

- si c'était la première fois qu'elle était suivie par les formateurs ou l'EN ;

- dans le cas où ce n'était pas la première fois, si elle avait été antérieurement enseignée par les formateurs ;

- dans le cas où ce n'était pas la première fois qu'elle était suivie par l'EN, si elle était en cours d'apprentissage ou faisait l'objet d'une interprétation.

Les chercheurs ont considéré que la règle était en cours d'apprentissage tant que l'EN s'engageait dans des suivis sans toutefois parvenir à constater les résultats attendus y étant usuellement associés. Ils ont considéré que la règle faisait l'objet d'une interprétation lorsque, suite à son apprentissage, un ou plusieurs éléments d'étayage étaient modifiés.

\section{Résultats}

Compte tenu de l'objet de cette étude, deux résultats significatifs sont présentés en suivant. Le premier résultat permet de défendre le postulat selon lequel la satisfaction professionnelle des EN est tout d'abord alimentée par l'engagement des formateurs dans une activité de formation exhaustive (constituée d'un enseignement ostensif des règles au formé, de l'accompagnement de leurs premiers suivis et éventuellement d'explications ostensives) afin de leur faire apprendre de nouvelles règles. Dans ces circonstances de formation, la satisfaction apparaît alors comme consubstantielle au constat par les EN des 


\section{eJRIEPS 31 janvier 2014}

résultats attendus suite aux suivis de ces nouvelles règles préalablement enseignées mais non encore apprises. Le second résultat permet de défendre le postulat selon lequel la satisfaction professionnelle des EN est d'autre part alimentée par l'engagement des formateurs dans une activité de formation les accompagnant dans l'interprétation de règles préalablement apprises et déjà suivies de façon insatisfaisante en situation de travail.

4.1. Accroître la satisfaction d'EN en difficulté : Importance de l'accompagnement par les formateurs des premiers suivis des règles enseignées

Au cours de l'EAC relatif à la leçon de la Visite Formative 3, l'EN de la Triade 1 a interpellé le chercheur sur le fait que, pour la première fois, elle a trouvé satisfaction (« je me dis : enfin! Ouf, ça y est, c'est bon on y est arrivé ") à suivre les conseils des formateurs (Extrait 1).

Extrait 1:

EN : Là, vous voyez, ça marche. Ils (les élèves) écoutent.

Chercheur $(\mathrm{CH})$ : Qu'est-ce qui marche là exactement ?

EN : Ben en fait là je baisse ma voix, et hop ça va vite, ils s'en rendent compte et hop ils s'arrêtent (de bavarder)...

$\mathrm{CH}$ : Et donc tu me dis...

EN : Ben là c'est quand même un soulagement. Dans ma tête là je me dis : « enfin ! Ouf, ça y est, c'est bon on y est arrivé ».

$\mathrm{CH}$ : Ouais ça a l'air de te soulager...

EN : Ouais complètement... Ca a mis du temps mais là voilà on y est (...)

$\mathrm{CH}$ : Mais précisément là, exactement en fait tu cherchais à faire quoi... Enfin je veux dire là ce que tu n'arrivais pas à faire, c'est quoi finalement ?

EN : Ben là c'est un truc qu'on (avec les formateurs) bosse en fait depuis le début (du dispositif d'aide). L'idée c'est pour contrôler, on joue le silence. C'est un peu ça l'idée en fait.

$\mathrm{CH}:$ C'est-à-dire ?

EN : Ben pour les faire écouter moi je montais (la voix) si vous voulez, de plus en plus, et eux en fait finalement ils montaient avec moi. Donc l'idée c'était de moins le faire au contraire. Mme Renaud (la FU) m'a proposé de parler moins fort pour justement les obliger en fait à être plus attentifs... Ils écoutent plus en fait si je parle moins fort... Et là c'est, c'est ce qui se passe en fait. Ils écoutent plus... donc voilà je les contrôle mieux, c'est ce que je dois travailler. 
eJRIEPS 31 janvier 2014

$\mathrm{CH}$ : Et donc là si je comprends bien aujourd'hui c'est la première fois que ça marche en fait?

EN : Ouais. D'habitude c'est quand même pas aussi efficace...

Cet extrait permet de documenter deux principales idées. Tout d'abord, l'EN trouve satisfaction suite aux suivis d'une règle qui lui a été préalablement enseignée par la FU (“Contrôler les élèves » vaut pour « jouer sur le silence » c'est-à-dire " parler moins fort aux élèves » ce qui obtient comme résultat qu'ils sont " plus attentifs " et "écoutent »). Plus précisément, l'EN trouve satisfaction dans la situation d'enseignement considérée, car elle parvient en suivant la règle à constater les résultats que lui avait associé la FU lors de son enseignement ("ça marche. Ils écoutent », "voilà on y est », "Et là ça y est (...) ils écoutent plus"). Complémentairement, cet extrait permet de situer qu'il a fallu du temps ("ça a mis du temps ", "D'habitude c'est quand même pas aussi efficace... ») à l'EN pour parvenir à être satisfaite suite au suivi de la règle considérée. Autrement dit, il a donc fallu l'engagement répété de l'EN dans des suivis de la règle préalablement enseignée par la FU pour parvenir à en constater les résultats attendus et finalement clôturer son apprentissage.

A ce niveau de développement se pose la question relative à la nature de l'activité de formation engagée par la FU et la T pour parvenir à cet apprentissage de la règle et par-là même à l'accroissement de la satisfaction de l'EN. L'extrait qui suit, emprunté à l'entretien de conseil pédagogique réalisé lors de la Visite Formative 1 (trois semaines plus tôt), permet d'illustrer l'engagement de la FU dans une activité d'enseignement ostensif de la règle considérée (Extrait 2).

\section{Extrait 2 :}

FU : T'as vraiment pas intérêt à essayer de toujours parler comme ça, parler, parler toujours de plus en plus fort. Alors je sais pas si tu t'en rends compte mais vraiment tu finis par vraiment crier quoi (...). Donc si tu veux par rapport à ça, parce que là on est en plein sur ce qu'on essaye de travailler quand même. Là ça permet de mieux contrôler la classe quand même... donc pour moi, après Isabelle (la T) te dira certainement ce qu'elle en pense, ou elle te l'a peut-être déjà dit, mais pour moi c'est un peu l'inverse... Moi je t'inviterais à faire l'inverse en fait. Gagner le silence par le silence si tu veux. L'idée c'est jouer sur la voix finalement. Tu réduis... Et ils (les élèves) vont être obligés de s'arrêter (de bavarder), de tendre l'oreille à ce que tu dis.

EN : Oui, oui mais c'est pas... 


\section{eJRIEPS 31 janvier 2014}

FU : Oui pas simple oui. C'est sûr mais... c'est quand même efficace. Tu baisses la voix jusqu'à ce qu'ils t'écoutent. Tu les obliges en quelque sorte à être attentifs...

La difficulté rencontrée par l'EN pour apprendre la règle considérée ne semble pas dépendante d'un engagement des formatrices dans une activité de formation inadaptée. A ce moment de l'entretien de conseil pédagogique, la FU s'engage en effet dans une activité d'enseignement ostensif de la règle. Elle dresse un lien de signification entre, d'une part, l'énoncé de cette dernière ("Contrôler la classe») et, d'autre part, des circonstances expérientielles décrites ("jouer sur la voix», "baisser la voix»). Elle précise par ailleurs les résultats qu'il est possible d'attendre suite au suivi de cette règle ("les élèves vont être obligés de s'arrêter de bavarder ", "d'être attentifs »). En ce sens, la FU rend possible un engagement de l'EN dans des premiers suivis de la règle avec des attentes, c'est-à-dire un engagement susceptible d'alimenter sa satisfaction professionnelle.

A ce niveau de développement, la question qui subsiste est celle relative aux circonstances qui ont finalement contribué à l'apprentissage de la règle considérée lors de la leçon de la Visite Formative 3. L'extrait d'EAC de la T relatif à cette leçon permet d'y répondre (Extrait 3).

Extrait 3 :

T : Là c'est comme tout à l'heure. Je sais pas si tu as vu mais bon à chaque fois pour les consignes, à chaque fois qu'elle donne des consignes avec Dominique (la FU) on se fait plus présentes. Tout à l'heure d'ailleurs Dominique a même changé de place...(...)

$\mathrm{CH}$ : C'était donc prévu de l'aider comme ça.

$\mathrm{T}$ : Oui là par rapport aux fois d'avant (les leçons antérieures) on (avec la FU) avait dans l'idée de lui simplifier ces moments où elle doit délivrer les consignes. Donc on se sépare, moi je me suis à un moment donné assise à côté des deux trois (élèves) agités... Mais on le lui (à l'EN) avait dit quand même. On lui avait demandé ça, si on pouvait un peu se montrer.

Cet extrait permet de situer que ce n'est pas seulement parce que l'EN s'est engagée dans des premiers suivis répétés de la règle que lui avait préalablement enseignée la FU qu'elle parvient tout à coup à clôturer son apprentissage et à s'en trouver satisfaite. L'engagement des formateurs dans une activité d'accompagnement de ces suivis a aussi contribué à ce résultat. Contrairement à ce qu'elles faisaient jusqu'à présent, les formatrices ont cherché lors de la leçon considérée à aménager la situation de travail de 


\section{eJRIEPS 31 janvier 2014}

l'EN. Plus précisément, elles ont essayé de "simplifier » à l'EN les temps de passation des consignes en s'efforçant d'être "plus présentes". En se positionnant différemment dans la classe (" on se sépare ", " je me suis à un moment donné assise à côté des deux trois (élèves) agités»), les formatrices ont limité l'agitation des élèves. Elles ont par làmême permis à l'EN de s'engager dans des suivis de la règle préalablement enseignée mais aussi et surtout d'en constater les résultats attendus. Par leur engagement dans une activité de formation constituée d'un enseignement ostensif de la règle en situation de formation puis de l'accompagnement effectif de ses premiers suivis en situation de classe, les formatrices ont donc contribué à l'apprentissage de l'EN et alimenté sa satisfaction professionnelle.

4.2. Accroître la satisfaction d'EN en difficulté : importance de l'accompagnement par les formateurs de l'interprétation de règles apprises

Au cours de l'EAC relatif à la leçon de la Visite Formative 2, l'EN de la Triade 2 a interpellé le chercheur sur le fait que les conseils des formatrices lui ont permis d'être un peu plus "efficace». A l'instant considéré de la leçon, elle est en effet d'autant plus satisfaite (" c'est plutôt bien là", "ça marche quand même pas mal») de son activité que les élèves ont pour la plupart réalisé le travail (« ils ont quand même tous bossé ») qu'elle leur avait donné (Extrait 1).

\section{Extrait 1:}

EN : Là, ouf, c'est plutôt bien là. Y a quand même ce moment-là. C'est ce que je vous disais tout à l'heure. Ils ont quand même tous bossé. Donc c'était dur... C'était pas. Pour moi avant là c'était dur. Mais là sur le temps où on essaye de faire la synthèse, là au tableau. Pour moi là, oui un espèce de ouf ça marche quand même pas mal donc c'est...

$\mathrm{CH}$ : Oui c'est...

EN : C'est pas mal là... Là je dirais que c'est le meilleur moment-là. J'ai l'impression d'être un peu plus efficace là...

Invitée par le chercheur à davantage expliciter les circonstances singulières de classe ayant conduit à cette satisfaction, l'EN précise en quelque sorte l'historique du travail de formation mené avec les formatrices sur la règle considérée (Extrait 2).

\section{Extrait 2 :}

$\mathrm{CH}$ : Le temps de recherche vous l'aviez vu je ne me souviens pas de ça tu peux me préciser? 
eJRIEPS 31 janvier 2014

EN : Oui... Si vous voulez ce que je faisais moi c'était de faire comme on avait vu en fait avec Isabelle (la $\mathrm{T}$ ). Je les (les élèves) mettais au travail et je puis après je les interrogeais... Avec quand même l'idée d'alterner en fait. J'interrogeais un élève studieux disons et puis juste après un autre mais là plutôt, plus agité. Et comme ça bon on avançait quand même et ils étaient plus attentifs... Pendant les exercices j'essayais de tenir la classe comme ça en fait...

$\mathrm{CH}$ : Donc ça c'était avant et là donc aujourd'hui tu as changé ?

EN : Ben là en fait... Comme moi, en fait, quand je faisais ça je trouvais que certains ne travaillaient pas vraiment en fait. C'était pas complètement satisfaisant en fait... Donc après on en a parlé quand elles l'ont vu et elles (les formatrices) m'ont proposé de faire aussi attention au travail de recherche. D'être en fait surtout avec certains élèves avant... quand ils essayent de répondre. Et c'est ça que j'ai fait là mais c'était dur quand même...

Lors du travail de formation engagé avec la T, l'EN avait appris la règle suivante : "Tenir la classe pendant les exercices » vaut pour " interroger par alternance les élèves studieux et les élèves agités » ce qui obtient comme résultat de rendre les élèves " plus attentifs " et d'« avancer » dans le travail. Lors du suivi de cette règle en classe, elle n'était toutefois pas « complètement satisfaite » du travail de l'ensemble des élèves («certains (élèves) ne travaillaient pas vraiment en fait.»). Elle a donc profité de l'entretien de conseil pédagogique de la Visite Formative 1 pour interpeller les formatrices sur cette difficulté. Ces dernières lui ont alors proposé une interprétation possible de la règle considérée en élargissant les aspects la constituant ("faire aussi attention au travail de recherche ») afin qu'elle soit davantage satisfaite à l'occasion des suivis de cette dernière.

L'extrait qui suit est justement emprunté à l'entretien de conseil pédagogique réalisé lors de la Visite Formative 1 (deux semaines plus tôt). II est doublement illustratif. D'une part, il permet de documenter l'idée selon laquelle le manque de satisfaction de l'EN, suite aux suivis dans certaines circonstances de classe d'une règle préalablement apprise, est à l'origine du questionnement qu'elle adresse aux formatrices. D'autre part, il permet de mettre en évidence l'engagement collectif de la $\mathrm{FU}$ et de la $\mathrm{T}$ dans une activité d'interprétation de la règle considérée (Extrait 3).

Extrait 3 :

$\mathrm{T}$ : Pour moi, le moment des exercices c'est nettement mieux. Ils (les élèves) sont beaucoup plus avec toi. Enfin, moi je trouve que c'est à noter quand même. 
eJRIEPS 31 janvier 2014

EN : Oui, en même temps. C'est pas, c'est pareil pour tous. Malika par exemple. Et sa copine là, euh, Solenne. J'ai l'impression que ce sont en fait toujours les mêmes, quoi que je fasse eux...

FU : Ils te dérangent. Mais ce que vous avez décidé à mon avis c'est quand même intéressant. Parce qu'en fait en changeant comme ça quand tu les questionnes ils sont obligés d'être un peu attentifs. Donc quelque part ça les tient.

EN : Oui c'est sûr, c'est mieux... Quand j'interroge c'est vrai ils (les élèves) sont un peu tenus mais... pour autant moi ce qui me gène c'est qu'ils n'ont vraiment pour certains travaillé le truc en fait. C'est moi souvent. Je réponds en fait, à leur place.

FU : C'est plus le travail en fait qui te... plus que le contrôle j'ai l'impression maintenant qui te...

EN : Oui. Moi je suis pas sûre qu'ils aient vraiment travaillé en fait. C'est quand même moi qui réponds en fait quand même là, pour certains.

Cet extrait permet de mettre en évidence que, insuffisamment satisfaite par les résultats obtenus suite au suivi d'une des règles apprises avec la $T$, l'EN rentre en controverse avec le point de vue porté par cette dernière sur l'activité des élèves lors du temps de la leçon consacré aux exercices. Alors que la $T$ considère que " c'est nettement mieux » en terme d'investissement des élèves à ses côtés, l'EN relève que ce jugement ne peut être généralisé à toute la classe ("c'est pareil pour tous »). En précisant par ailleurs qu'elle est démunie de solutions (" quoi que je fasse ») pour répondre à la difficulté qu'elle rencontre ( "je suis pas sûre qu'ils aient vraiment travaillé », "C'est quand même moi qui répond »), elle sollicite indirectement l'aide des formatrices. C'est ce que confirme l'extrait de son EAC proposé ci-après. Lors de cet extrait, elle insiste en effet sur le caractère encore non complètement satisfaisant des résultats obtenus ("ce qu'on (avec la $T$ ) avait décidé ne marche pas tout le temps") suite aux suivis de la règle considérée. Elle s'engage aussi dans une explicitation de ce qu'elle a pu constater lors de la leçon avec comme intention première d'engager la discussion sur ce point ("je voudrais qu'on en discute ») et faire en sorte que des solutions soient trouvées pour suivre la règle de « façon peut être différente avec certains » élèves (Extrait 4).

\section{Extrait 4 :}

EN : Là je reviens sur le fait que ce qu'on (avec la T) avait décidé ne marche pas tout le temps en fait.

$\mathrm{CH}$ : Vous n'êtes pas d'accord on dirait? 


\section{eJRIEPS 31 janvier 2014}

EN : Je sais pas. Là je voudrais qu'on en discute. OK alterner quand je questionne c'est pas mal mais pas pour tous (les élèves).

$\mathrm{CH}$ : Donc tu leur dis ça?

EN : Je dis ce que je ressens en fait. Ce que je vois que peut être eux n'ont pas vu.

$\mathrm{CH}$ : C'est exprès là. Elle dit c'est bien et toi tu dis non pas tout à fait. Tu...

EN : Non moi là c'est pour mieux faire en fait. Le faire de façon peut-être différente avec certains (élèves).

Lors de son EAC relatif à cette séquence de formation, la FU suit la règle suivante : "Enrichir la pratique de l'EN » vaut pour « confirmer que ce qui a été fait fonctionne » et " essayer de l'adapter pour chaque situation particulière » ce qui obtient comme résultat de « ne pas remettre tout en cause » et " ne pas déstabiliser l'EN". A l'instant considéré, son intention n'est donc pas de faire apprendre de nouvelles règles à l'EN mais plutôt de l'aider à "adapter" les suivis de règles préalablement apprises à la singularité des circonstances de classe. II est donc possible de considérer que la FU mène une activité de formation qui vise à soutenir le développement professionnel de l'EN en accompagnant son interprétation de règles préalablement apprises (Extrait 5).

Extrait 5 :

FU : Ce qu'on fait là c'est pour pas. On ne veut pas tout remettre en cause en fait.

$\mathrm{CH}$ : Remettre en cause. C'est-à-dire ?

FU : Ce qu'elle fait n'est quand même pas complètement, complètement inefficace. Donc pour pas trop la déstabiliser on cherche plutôt en enrichir ce qu'elle fait. On va pas tout changer quoi.

$\mathrm{CH}$ : Et l'enrichir là pour toi c'est quoi là?

FU : Ben là on essaye en fait de... Bon déjà de confirmer que ce qui a été fait fonctionne si elle risque de... Déjà. Et puis, après on essaye de trouver un moyen pour l'adapter.

Lors de son EAC relatif à cette même séquence de formation, la T confirme qu'elle a, elle aussi, pour intention "d'améliorer ce que fait déjà " de façon satisfaisante l'EN. Complémentairement aux propos tenus par la FU, la $\mathrm{T}$ précise l'interprétation qu'elle cherche à engager avec l'EN à propos de la règle qu'elle avait elle-même enseignée. En avançant une "solution qui complète " ce qui était fait jusqu'à lors, elle cherche à amener l'EN à suivre la même règle tout en l'interprétant comme suit : "Tenir la classe pendant les exercices " vaut pour " préparer les réponses aux exercices avec les élèves agités " et «interroger par alternance les élèves studieux et les élèves agités » ce qui obtient 


\section{eJRIEPS 31 janvier 2014}

comme résultat de rendre les élèves "plus attentifs » et d'« avancer » dans le travail. L'extrait d'EAC de la T qui suit documente ces idées (Extraits 6).

\section{Extrait 6:}

$\mathrm{CH}$ : Là, toi tu proposes une solution en fait? C'est la première fois que tu interviens comme ça?

$\mathrm{T}$ : Oui, mais c'est une solution pour compléter en fait, là. Je complète ce qui est dit par Béatrice (la FU)

$\mathrm{CH}$ : C'est mieux donc.

$\mathrm{T}$ : Oui nettement. Mais là c'est plutôt essayer d'améliorer en fait ce qui a été fait puisqu'elle (l'EN) nous dit que bon pour certains c'est pas encore bien, bien concluant en fait... Par rapport au travail.

$\mathrm{CH}$ : Et donc toi là tu?

$\mathrm{T}$ : Ben là pour lui répondre en fait. On... je lui dis t'as qu'à bosser directement avec eux (les élèves) quoi comme ça tu es sûre de...

$\mathrm{CH}$ : Bosser avec eux, c'est-à-dire...

$\mathrm{T}$ : Oui elle peut peut-être avant de faire ce type d'échanges là, comme là aujourd'hui, elle peut peut-être travailler directement avec eux. Avant, pour préparer les réponses directement avec eux. Là ils travailleront si elle les fait vraiment travailler.

Finalement, par leur engagement dans une activité de formation accompagnant l'interprétation de règles préalablement apprises et déjà suivies de façon insatisfaisante en situation de travail par l'EN, les formatrices ont donc à la fois contribué à l'accroissement de la satisfaction de cette dernière ainsi qu'à son développement professionnel.

\section{Discussion et conclusion}

Cette discussion ne cherche pas à généraliser des résultats issus d'une étude de cas. Elle vise seulement à mettre en exergue les circonstances de formation, explicitées dans les résultats, ayant sans doute contribué à l'apprentissage de nouvelles règles par l'EN mais aussi à l'accroissement de sa satisfaction professionnelle.

Dans leur ensemble, les résultats de notre étude permettent de discuter de l'efficacité du principe de l'alternance tel qu'il est usuellement exploité en formation professionnelle des enseignants. Ils permettent plus précisément d'interroger le nécessaire aménagement des situations de travail, en véritables situations de formation «au travail par le travail» (Durand \& Fillietaz, 2009), afin de réduire l'étanchéité constatée (Nir et al., 2008) entre les 


\section{eJRIEPS 31 janvier 2014}

séquences de formation et les séquences de travail et in fine de concourir à davantage de satisfaction professionnelle des EN au travail. Dans le détail, et même s'ils appellent à être confirmés, nos résultats permettent de tracer deux directions pour engager cet aménagement du principe de l'alternance en formation. Tout d'abord, les situations de travail devraient sans doute être davantage aménagées pour faciliter la finalisation de l'apprentissage des règles préalablement enseignées. L'idée serait alors d'aménager les situations de travail afin que les EN puissent s'engager dans des premiers suivis des règles qui leur ont été enseignées et constater les résultats qui leur sont usuellement associés. D'autre part, les situations de travail devraient aussi être aménagées afin de permettre aux EN d'interpréter plus facilement des règles déjà apprises et de s'en trouver ici aussi satisfaits. Autrement dit, les formateurs pourraient alors les accompagner dans une émancipation progressive de ces dernières. Ces deux aménagements permettraient de placer les EN dans des conditions de formation par le travail, conditions facilitantes parce que leur permettant de suivre les règles préalablement enseignées ou apprises jusqu'à en constater les résultats attendus, c'est-à-dire jusqu'à s'en trouver satisfaits professionnellement.

Fort de ces deux propositions, on comprend dès lors toute l'importance de penser autrement l'accompagnement de la formation par les formateurs. En rendant viable des conditions d'exercice du métier trop souvent difficiles, ce type d'accompagnement par les formateurs de la pratique professionnelle des EN accentue tout à la fois les retombées sur leur formation mais aussi leur satisfaction (Mtika et al., 2011). En créant un climat social positif, les formateurs contribuent en effet de façon effective à accroître la satisfaction des EN (Day et al., 2007). Plus précisément encore, la complémentarité des T et des FU apparaît comme fondamentale dans cet accompagnement des EN. Les étiquetages des règles par les FU nécessitent en effet une activité d'exemplarisation par et sur des échantillons de leçon des EN par les T afin d'en faciliter a posteriori leurs suivis. Les FU connaissent autrement dit les règles génériques de métier indispensables à la construction de l'identité professionnelle des EN (Roux-Perez, 2010). L'activité d'enseignement ostensif de ces règles semble donc plutôt de leur responsabilité. Par contre, compte tenu de leur expérience professionnelle et de leur connaissance du contexte d'enseignement des EN, les tuteurs connaissent tout à la fois les exemples les plus adaptés pour leur permettre ensuite un suivi adéquat en classe des règles enseignées mais aussi les circonstances de travail pouvant autoriser ce suivi. Autrement dit, les activités « d'échantillonnage » 


\section{eJRIEPS 31 janvier 2014}

(Williams, 2002) des règles enseignées et d'aménagement des situations de travail pour en autoriser le suivi apparaissent donc comme de la responsabilité des $T$.

Nos résultats montrent en outre que ce type d'accompagnement des EN par les formateurs permet d'aider davantage à la contextualisation des suivis des règles en contexte classe. Thompson, Hallwood, Clements \& Rivron (2009) relèvent en effet que les EN rencontrent des difficultés pour suivre de façon adaptée et circonstanciée dans leur établissement respectif les règles qui leur ont été préalablement enseignées de façon générique à l'université. Même si des efforts de contextualisation sont effectués en formation, ils se révèlent généralement bien moins efficaces que l'aide directement fournie par les formateurs en contexte classe (Chaliès, Escalié, Cartaut \& Durand, 2009). Ce n'est donc qu'à travers ce travail d'accompagnement et de suivis des EN en situation d'exercice du métier, que les formateurs semblent pouvoir contribuer tout autant à leur formation qu'à leur satisfaction professionnelle.

\section{Références}

Berducci, D. (2004). Developmental Continuum Vygotsky through Wittgenstein: A New Perspective on Vygotsky's. Theory Psychology, 14, 329-353.

Bertone, S., Chaliès, S., \& Clot, Y. (2009). Contribution d'une théorie de l'action à la conceptualisation et à l'évaluation des pratiques réflexives dans des dispositifs de formation initiale des enseignants. Le Travail Humain, 72, 104-125.

Bullough, R., Bullough, D. A. M, \& Mayes, P. B. (2006). Getting in touch: dreaming, the emotions and the work of teaching. Teachers and Teaching: Theory and Practice, 12(2), 193-208.

Caprara, G. V., Barbaranelli, C., Steca, P., \& Malone, P. S. (2006). Teachers' self-efficacy beliefs as determinants of job satisfaction and students' academic achievement. Journal of School Psychology, 44, 473-490.

Cash, M. (2009). Normativity is the mother of intention: Wittgenstein, normative practices and neurological représentations. New ldeas in Psychology, 27, 133-147.

Chaliès, S., Bertone, S., Flavier, E., \& Durand, M. (2008). Effects of collaborative mentoring on the articulation of training and classroom situations: A case study in the French school system. Teaching and Teacher Education, 24(3), 550-563. 


\section{eJRIEPS 31 janvier 2014}

Chaliès, S., Escalié, G., Cartaut, S., \& Durand, M. (2009). L'utilité du tutorat pour de jeunes enseignants : la preuve par 20 ans d'expérience. Recherches et formation, 61, 85-129.

Chauviré, C. (Ed.). (2010). Wittgenstein en héritage. Philosophie de l'esprit, épistémologie, pragmatisme. Paris : Kimé.

Clot, Y. (Ed.). (2010). Le travail à cœur. Pour en finir avec les risques psychosociaux. Paris : La Découverte.

Davis, A. (2009). Examples as method? My attempts to understand assessment and fairness in the spirit of the later Wittgenstein. Journal of Philosophy of Education, 43, 371-389.

Day, C., Sammons, P., Stobard, G., Kington, A., \& Gu, Q. (2007). Teachers matter: Connecting work, lives and effectiveness. Berkshire, England: Open University Press.

Demirtas, Z. (2010). Teachers' job satisfaction levels. Procedia Social and Behavioral Sciences, 9, 1069-1073.

Descombes, V. (2004). Le complément de sujet. Enquête sur le fait d'agir soi-même. Paris: Gallimard.

Dinham, S. (1995). Time to focus on teacher satisfaction. Unicorn, 21(3), 64-75.

Duffy, R. D., \& Lent, R. W. (2009). Test of a social cognitive model of work satisfaction in teachers. Journal of Vocational Behavior, 75, 212-223.

Durand, M., \& Filliettaz, L. (2009) Travail et formation des adultes. Paris: Presses universitaires de France.

Fabra, M.E., \& Camison, C. (2009). Direct and indirect effects of education on job satisfaction: A structural equation model for the Spanish case. Economics of Education Review, 28, 600-610.

Francès, R. (1995). Motivation et efficience au travail. Liège: Mardaga.

Judge, T.A, Thoresen, C.J, Bono, J.E., \& Patton, G.K. (2001). The Job Satisfaction-Job Performance Relationship: A Qualitative and Quantitative Review. Psychological Bulletin, 127(3), 376-407.

Kyriacou, C. (2001). Teacher stress: Directions for future research. Educational Review, 53(1), 27-35.

Laugier, S., \& Chauviré, C. (2006). Lire les recherches philosophiques de Wittgenstein. Paris: Vrin. 
eJRIEPS 31 janvier 2014

Lave, J., Wenger, E. (Eds.). (1991). Situated learning: Legitimate peripheral participation. Cambridge: Cambridge University Press.

Leung, T., Siu, O. \& Spector, P. (2000). Faculty stressors, job satisfaction, and psychological distress among university teachers in Hong Kong: The role of locus of control. International Journal of Stress Management, 7(2), 121-138.

Liu, X. S. \& Ramsey, J. (2008). Teachers' job satisfaction: Analyses of the Teacher Follow-up Survey in the United States for 2000-2001. Teaching and Teacher Education, 24, 1173-1184.

Moè, A., Pazzaglia, F. \& Ronconi, L. (2010). When being able is not enough. The combined value of positive affect and self-efficacy for job satisfaction in teaching. Teaching and Teacher Education, 26(5), 1145-1153.

Mtika, P., \& Gates, P. (2011). What do secondary trainee teachers say about teaching as a profession of their "choice" in Malawi? Teaching and Teacher Education, 27, 424-433.

Narboux, J.P. (2003). Incommensurabilité et exemplarité : aliénation logique et problème des universaux. Archives de Philosophie, 66, 437-447.

Nelson, K. (2008). Wittgenstein and contemporary theories of word learning. New ideas in psychology, 4(3), 1-13.

Nir, A.E. \& Bogler, R. (2008). The antecedents of teacher satisfaction with professional development programs. Teaching and Teacher Education, 24, 377-386.

Ogien, A. (2007). Les formes sociales de la pensée: la sociologie après Wittgenstein. Paris: Armand Collin.

Rogoff, B., Matusov, E., \& White, C. (1996). Models of teaching and learning: participation in a community of learners. In D.R. Olson \& N. Torrance (Eds.), The handbook of education and human development: New models of learning, teaching and schooling (pp. 388-414). Oxford: Basil Blackwell Publishers.

Roux-Perez, T. (2010). Quelle prise en compte du Sujet dans la formation des enseignants ? Enjeux et limites d'une approche clinique. In Baillat, G., Niclot, D., \& Ulma,D., (sdr.). La formation des enseignants en Europe (pp. 127-138). Bruxelles : De Boeck.

Shen, J., Leslie, J.M., Spybrook, J.K., \& Ma, X. (2011). Are Principal Background and School Process Related to Teacher Job Satisfaction? A Multilevel Study Using Schools and Staffing Survey 2003-04. American Educational Research Journal, 48(5), 1-31. 


\section{eJRIEPS 31 janvier 2014}

Skaalvik, E.M., \& Skaalvik, S. (2010). Teacher self-efficacy and teacher burnout: a study of relations . Teaching and Teacher Education, 26, 1059-1069.

Smylie, M.A. (1994). Redesigning Teachers' Work: Connections to the Classroom. Review of Research in Education, 20, 129-177.

Thompson, R., Hallwood, L., Clements, C., \& Rivron, H. (2009). Personal development planning in initial teacher training: a case study from post-compulsory education. Research in Post-Compulsory Education, 14(3), 269-285.

Weiss, H.M. (2002). Deconstructing job satisfaction: separating evaluations, beliefs and affective experiences. Human Resource Management Review, 22, 173-194.

Williams, M. (2002). Tout est-il interprétation ? In C. Chauviré \& A. Ogien (Eds.), La régularité (pp. 207-233). Paris : EHESS.

Winch, P. (Ed.). (2009). L'idée d'une science sociale et sa relation à la philosophie. Paris : Gallimard.

Wittgenstein, L. (2004). De la certitude. Paris : Gallimard. 